\title{
ASSESSING JOB MARKET DYNAMICS USING ELK STACK
}

\author{
Gabriel Silva $^{1}$, Mário Rodrigues ${ }^{2}$, Marlene Amorim $^{3}$, Angélica Souza ${ }^{4}$, Marta Dias $^{3}$ \\ and Armando Pinho ${ }^{5}$ \\ ${ }^{1}$ DETI, ${ }^{2}$ ESTGA \& IEETA, ${ }^{3}$ DEGEIT \& GOVCOPP, ${ }^{4}$ DEGEIT, ${ }^{5}$ DETI \& IEETA \\ University of Aveiro, Campus Universitário de Santiago, Aveiro, Portugal
}

\begin{abstract}
The adoption of digital technologies promises to accelerate the transformation and the agility of processes, work activities and revenue models. Yet, the promised gains come together with dramatic needs for qualified professionals who can effectively leverage the technology potential. Job contexts are being reshaped as new models for the interaction and integration of humans and technologies take shape.

To increase the readiness of the job market in this fast-changing context it is important that all stakeholders - companies, professionals, policy makers - are aware of the job market dynamics and needs. This can be observed from the collection of job announcements, but its high volume requires effective tools for analyzing and simplifying it in order to draw timely and correct conclusions.

ELK stack was used for dealing with the high volume of job announcements. ELK is a stable platform that can manage large quantities of data and the Kibana layer enables to rapidly explore data and create visualization dashboards. As job announcements have distinct formulations for similar roles, depending on the hiring company, this raises the necessity of establishing a common ground for comparing the job descriptions. In this work were mapped job descriptions to ESCO occupations. ESCO is an ontology published by the European Union and its occupations are job positions.

Results show that the ELK stack is a suitable tool for providing a visual interpretation on the job market dynamics. Moreover, the first experiments using natural language processing techniques and machine learning algorithms revealed an accuracy over 0.9 in mapping job descriptions to ESCO occupations. This result is very promising and shows that ESCO a good candidate as common ground to enable comparison of job market dynamics for distinct environments.
\end{abstract}

\section{KEYWORDS}

Natural Language Processing, Ontology, Data Overview, ESCO, ELK Stack, Job Market

\section{INTRODUCTION}

Digitalization is one of the most popular topics in the agendas of companies and policy makers. For companies, the adoption of digital technologies promises to accelerate the interconnectedness and the agility of processes, work activities and revenue models (Szalavetz, 2019). Yet, the promised gains come together with dramatic needs for qualified professionals who can effectively leverage the technology potential. Job contexts are being reshaped as new models for interaction and integration of human talent and technologies take shape, and as enormous volumes of data are generated at every operation (Freddi, 2018).

The demand for qualified professionals that can make sense of such accelerated and interconnected work systems is already evident, notably in the explosive growth of job announcements in social media and online platforms. The challenge lies in the fact that many of the professional profiles that are in growing demand require new qualifications, and unique combinations of technical and behavioural competences that are hard to find in the current job market (Chryssolouris et al., 2013, Jermans et al., 2020). Several of these new jobs, such as app developers, cloud computing specialists, data scientists, drone operators, data brokers, cyber security specialists, among many, were inexistent in the last decade. For both companies and professionals, this is a critical moment to make sense of the new professional profiles and demands that are emerging, in order to adjust recruitment and qualification and career strategies. 
The popularity of online media for recruitment, and the abundance of job postings announced every day, offers a rich setting to learn about the characteristics of the new professional profiles that are in demand to meet the digitalization across all business sectors (Briscariu, 2019, Gelpi, 2019). Moreover, the understanding in the changes taking place in workforce demand is critical for the society, in order to develop detailed knowledge about the forthcoming skills requirements and support the development of adequate education and training programs in a timely manner. This study offers a contribution in such direction and for that it uses state of the art information visualization tools such as the ELK stack (ELK 2020). The study develops tools that allow for the extraction and the analysis of large volumes of data from online job announcements, with the purpose of advancing in the understanding about the emerging demands for competences and skills. The study offers a contribution to bring together the rich information about emerging demand for professional profiles that is scattered across new job announcements, and the existing structured knowledge about skills, competences, qualifications and occupations relevant for the EU labor market that is organized in ESCO (ESCO 2020), the European Skills, Competences and Occupations classification system.

In the next section it is discussed the related work, in section 3 is described the system developed followed by the achieved results and discussion in section 4 . The document ends in section 5 with the relevant conclusions.

\section{RELATED WORK}

In this section literature related to either extracting job information from postings or resumes is presented as well as articles using frameworks such as ESCO.

In the article (Fernández-Sanz, Gómez-Pérez and Castillo-Martínez, 2017), the authors develop a framework called eSkills Match. The framework attempts to align the existing work done by the European Union with the European e-Competence Framework (e-CF), ESCO and the Information and Communication (ICT) Body of Knowledge (BoK) into a single model. This model uses e-CF as the reference framework, tries to relate the BoK directly with it. The authors also use ESCO and relate it with the e-CF framework through skills and knowledges. In the end the authors had a set of 10 ICT job profiles that were used to test the matching method with only 4 of these being present in the article. The authors identify the difficulties in matching different frameworks, requiring external validation from experts at times as well as the need to include more jobs.

In a second article (Chen, Zhang and $\mathrm{Niu}, 2018$ ) the authors attempt to extract information from resumes. The authors identify two steps needed to extract information from resumes which are not standardized neither in format nor layout. The first step takes as input a resume where the raw text is extracted followed by identifying the composition of each line and using adaptive segmentation in order to output semi-structured data comprised of several key-value pairs. To help generate this data the authors design a novel feature called "Writing Style". The second step is called "Resume Facts Identification" where the previously generated semi-structured data is taken as an input, passed through a multiple category text classifier and the result is structured output resume data containing facts about the resume that was used as an input. The results are compared with PROSPECT and CHM with this method beating both in almost every test done with the added benefit of needing less hours of annotations.

A different approach to extracting information from resumes and converting it into structured data can be seen in (Celik Ertuğrul et al, 2013). An ontology-driven algorithm is used to extract information and match it with concepts. The methodology is based on concept-matching and ontological rules so that semantic analysis and data parsing can be performed on the resumes. Extracting information such as experience, features and business/education information and converting it onto an ontology. The authors propose two algorithms that can be used to accomplish this task and test them on both English and Turkish résumés.

E-Recruitment systems try and give the best possible match between candidates and job descriptions, in this article (Ahmed et al, 2019), the authors propose a new approach to building these systems. The system created extracts the job description text and segments with the help of dictionaries (that are a combination of rules and lists). This segmentation then passes through a Natural Language Processing (NLP) pipeline (sentence splitting, part-of-speech tagging, named entities extraction and compound words extraction) they are then sent to the "Context Builder" and "Enrichment" processes before being stored in the Knowledge Base. This approach is compared with other information extraction software such as OpenCalais and Alchemy and beats them both by quite a margin in all the metrics used (precision, recall and F1) and tests done (job title and requirement extraction). 


\section{DEVELOPED SYSTEM}

Working with different data sources is always a challenging task. Every source has their own schema as well as attributes and naming conventions. A goal to create an adaptive system that could easily ingest data from any source was set at the beginning of this project. Instead of ingesting data from every Application Programming Interface (API) and adding the documents as they were onto the database system, the decision was to create a data model that these documents could be converted to. Figure 1 shows the flow of data in the system that was created.

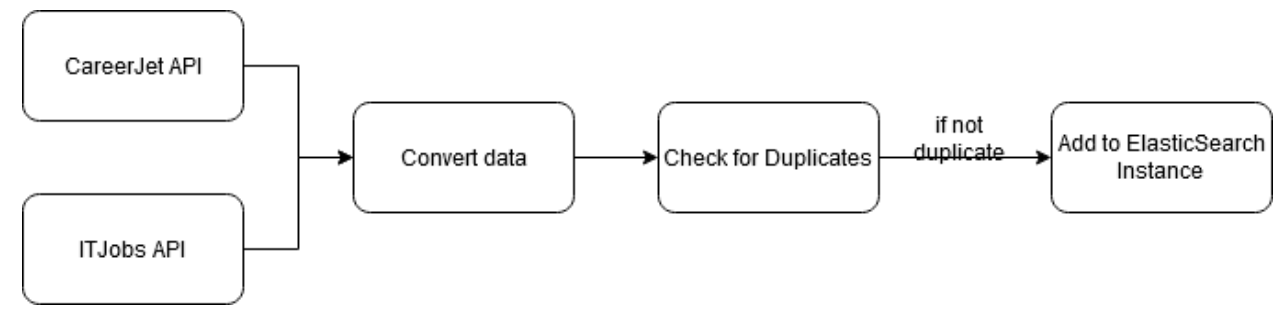

Figure 1. Data Information Flowchart

The creation of this shared data model allows for easier data crunching, inclusion of additional sources of data as well as duplicate detection since the model is the same. Overall, it allows for more control over the data and which fields can be used later for analysis. The whole system works with the ELK (ElasticSearch, Logstash, Kibana) stack (Bajer, 2017).

\subsection{Data Sources}

In order to develop this work, the first step taken was to find websites that had job offerings and see if there was an API provided so that data could be extracted and stored on our end. The websites considered were LinkedIn, CareerJet, ITJobs, Glassdoor, Stackoverflow, Indeed, Net-Empregos, and Sapo Empregos.

From these websites only CareerJet (CareerJet, 2020) and ITJobs (ITJobs, 2020) had APIs that provided listings to every job posted on their respective websites. LinkedIn also had an API that could be used; however, this would be against their terms of service meaning the accounts could be shut down at any time so it was not selected as a source for data extraction. Glassdoor, Stackoverflow and Indeed do have APIs available, however, there needs to be authorization for them to be used and to get an API key. The last two sources, Net-Empregos and Sapo Empregos, do not have an API that can be used and thus would require web-scrapping techniques in order to extract information. While this is an effective technique, changes on those websites could cause data extraction failures, which is not a desired outcome.

For early stage of development and testing just ITJobs and CareerJet are considered since they offer an API with direct access to the information displayed on their website. These websites offer information about a job posting such as: Posting Date, Company Information, Job Title, Job Description, Job Location among others.

Unfortunately, only ITJobs gives the complete Job Description which is the most useful thing, CareerJet cuts the Job description after a set number of characters making it so that web scrapping had to be used in conjunction with their API in order to get full descriptions and automate the whole process.

\subsection{Data Model}

Since every data source gives different fields with different names there was the decision to create a unified model under which it was possible to convert the results obtained from API calls to both ITJobs and CareerJet.

This Data model should be comprehensive and extensive but also easily expansible so that everything needed to feed both Kibana and the machine learning algorithms. It should also make use of all the information provided by each API. 
Looking at the data gathered by both ITJobs and CareerJet the data model ended up having the following 14 fields: (1) Source - Website it came from; (2) Location - Location of the job; (3) Date Posted - Date in which the job was posted in a year, month, day format; (4) Job Posting - Link to where the job was posted; (5) Title - Job title; (6) Description - The job description written by the company; (7) Company - Name of the recruiting company; (8) Website - Recruiting company website; (9) Contact - Recruiting company contact; (10) Allow Remote - If the work can be done remotely; (11) Salary - Salary ranged the company posted with the job; (12) Type - Whether it's a full-time job or part time; (13) Contract - If the job is temporary or not; and (14) Number of Words - Number of words the job description had.

Having created this model, it is now needed to convert and standardize the data obtained from querying both the ITJobs and the CareerJet APIs. The response obtained by the ITJobs (ITJobs, 2020) API did not need much conversion to our proposed data model. The API returns almost every field in our model (some with different names), except for "Source" and "Job Posting", however, there were incomplete postings that did not contain every field, with the one that was missing the most being "Salary". Besides having to fill in the fields that were not provided the date also had to be converted to the format used by our model.

CareerJet (CareerJet, 2020) was the more troublesome API. The job description had a fix number of characters after which the whole description was truncated. This was solved using web scrapping. The API returned the link to the job posting which is then used to extract the whole job description. Besides the trucated job descriptions the API does not return a lot of information that would be useful in our model. CareerJet is lacking the following fields: Contact, Type, Allow Remote, and Contract. Some of these might be possible to get in the future with more web scrapping or by using NLP methods on the job description.

\subsubsection{Handling Duplicates}

In order to consider several data sources, it was crucial that our data extraction algorithm could handle duplicates since most of the times companies don't post only on a single website but instead post to several. The first step towards achieving duplicates detection was to make both the job title as well as the company name lower-cased. This was done so that in case the websites handled things differently, for example, some websites like to capitalize the beginning of every word in the title. After standardizing titles and company names, the date a job was posted is also looked at as a possible duplicate detection. The month check is to account for companies that post jobs at different points in time, which could have a multitude of reasons behind, such as, they need mor people to fill that role or because they haven't found anyone within a month time frame which could be significant to identify a lack of specialized employees in that area. Since ElasticSearch uses the Lucene search engine behind, a score is attributed to documents based on their similarity to the query. Having no control over how this similarity score is calculated, in this algorithm, every document returned by the query is looked at as a possible duplicate.

In short, the algorithm is as follows:

\footnotetext{
Algorithm 1: Job posting duplicate detection

Query ElasticSearch for documents with the same title as the new job

For: Every document retrieved from the query:

If: New job posting title and company name do MATCH the job in the database then:

If: Month the new job was posted MATCHES the month of the job in the database then: Return True

Return False
}

In case the algorithm returns a "True" value this means that there is already a job posting in ElasticSearch with the same exact title, from the same company and posted in the same month so this document will not be added. However, in case the algorithm returns a "False" value this means that ElasticSearch could not find any document like the new one so it should be added.

\subsection{Data Presentation}

The data presentation layer is done through Kibana in combination with an ElasticSearch instance of ELK (ElasticSearch, Logstash, Kibana) stack. ElasticSearch indexes every field of the documents making it easy for Kibana to quickly get the information needed to generate the graphs. Kibana offers the ability to create 
several types of graphs and join them in a single interactive dashboard that can be queried. It is a powerful tool that allows users to create easy to read dashboards and compare, for example, the performance of two different cities in an activity sector.

Figure 2 presents an example of dashboard with four areas containing distinct views. Each view is interactive and selecting an attribute in one make the others refresh and adapt to the new filter(s).

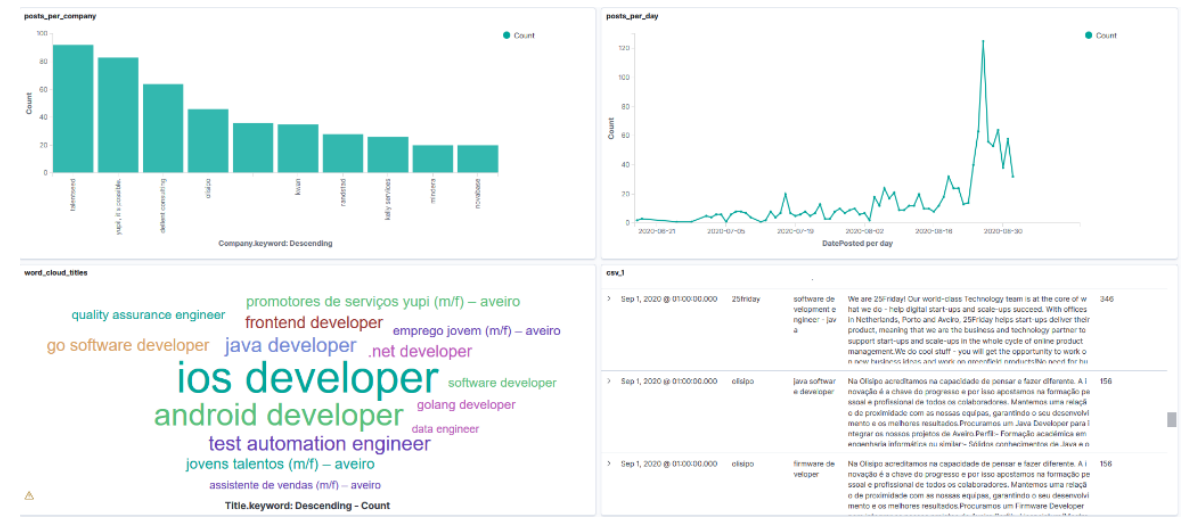

Figure 2. Example of a Dashboard

The dashboard allows an user to visualize the companies with the most job postings (top left bar graph), the number of job postings per day (top right graph), the most sought after job titles (bottom left) and a table visualization of the data that has date of the job posting, company, job title and recruitment description as well as number of words (bottom right).

With the same schema, these dashboards allow for users to select, for example, "ios developer" on the bottom left word cloud and the whole dashboards filters itself as seen in Figure 3.

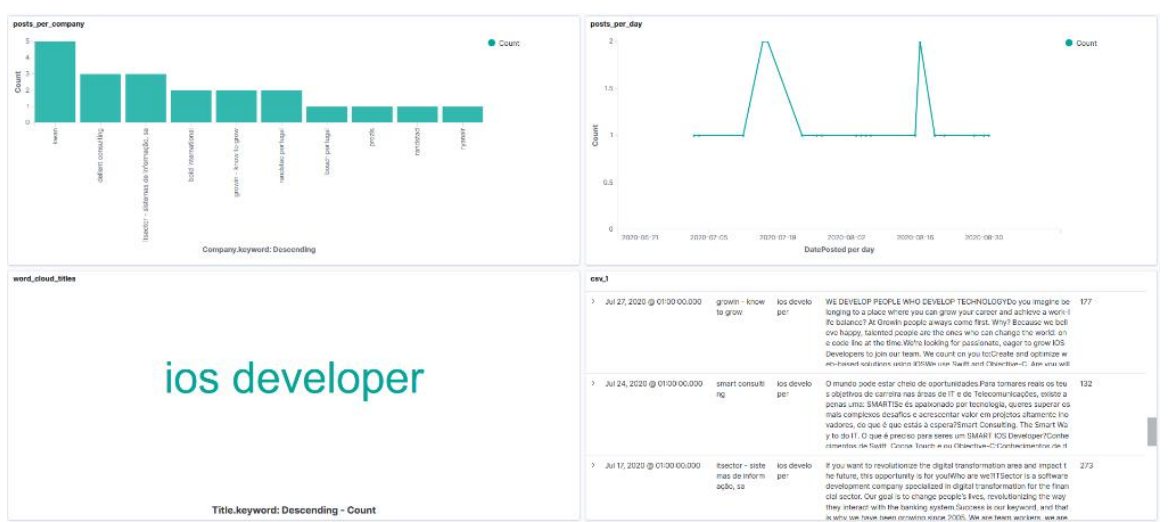

Figure 3. The updated dashboard when the user presses the term "ios developer" in the word cloud

\section{RESULTS AND DISCUSSION}

The system has been collecting data from ITJobs and CarrerJet for the past 5 months. We have limited the data collected to jobs proposals icluding the region of Aveiro, our region, just to not overload ITJobs and CarrerJet before we make sure that the developed system worked as designed. There was no optimization or specifically designed algorithm having this limitation into consideration. 


\subsection{Data}

So far there are close to four thousand data points (3920) in 5 months with more being added weekly. The filtering algorithm has excluded more than two thousand job postings with each job posting containing between 200-300 words per description with the minimum being 57 words and the maximum being 490 . When it comes to data sources, CareerJet has far more data, providing 3519 results while ITJobs has 409 results in our database. This discrepancy can be attributed to the fact that ITJobs is purely focused on job postings for IT roles.

Besides this there was also some pre-processing that had to be done. For our testing data we used a small sample from ITJobs, data corresponding to a week of postings. Some job descriptions had to be translated to English in order to maintain consistency with the rest of the offers.

\subsection{Ontology}

Each job gathered was classified as an ESCO occupation (European Skills, Competences and Occupations). ESCO describes 2942 occupations and 13485 skills linked to these occupations translated into 27 languages. The following protocol was implemented to train the algorithms to classify jobs as ESCO occupations. For each job posting that was gathered between days 11 and 18 of September 2020 an ESCO category was attributed manually. The manual classification was done by two people that independently classified each job posting, and a third person would choose a classification whenever the first two people did not agree. In the end, for the existing 58 job postings were assigned a total of 9 ESCO occupations, in which the jobs were classified as. However, the data was imbalanced with a few occupations having only 2 or 3 job postings associated with them while, for example, two other occupations have 16 and 14 job postings. Nevertheless, the experiment continued to assess if the approach is feasible and should be further investigated.

In Table 1 is visible an example of a job posting, with the description truncated, as well as the corresponding ESCO designation assigned to it. Table 2 shows the designations from ESCO as well as the labels representing each class used in the machine learning algorithm, alongside the number of samples for each designation.

Table 1. Example of a job announcement and the ESCO occupation assigned

\begin{tabular}{|c|c|c|}
\hline Title & Description & $\begin{array}{l}\text { ESCO } \\
\text { Occupation }\end{array}$ \\
\hline $\begin{array}{l}\text { Scala } \\
\text { engineer }\end{array}$ & $\begin{array}{l}\text { Keywords: Scala, Java, CI/CDYears of experience: } 3+\text { English level: Advanced ... we are } \\
\text { hiring Scala Engineer for a long term contract role in Porto, Aveiro SKILLS AND } \\
\text { EXPERIENCE:- Service-oriented architectures, design patterns and data structures;- Test } \\
\text { automation, continuous integration and continuous delivery;- Working with relational ... }\end{array}$ & $\begin{array}{l}\text { Software and } \\
\text { Application } \\
\text { Developers }\end{array}$ \\
\hline
\end{tabular}

Table 2. ESCO occupations used, the label for each occupation and the number of samples for each occupation

\begin{tabular}{lll}
\hline ESCO Designation & Label & Number of samples \\
\hline Software and Application Developers & 0 & 16 \\
\hline Analytics Specialist & 1 & 2 \\
\hline Back End Developer / Engineering & 2 & 5 \\
\hline Data Engineer & 3 & 2 \\
\hline DevOps Engineer / Cloud Computing & 4 & 4 \\
\hline Frontend Engineer / Engineering & 5 & 14 \\
\hline Hardware/Infrastructure Engineer & 6 & 3 \\
\hline Javascript Developer / Engineering & 7 & 8 \\
\hline Telecom specialists & 8 & 4 \\
\hline
\end{tabular}

After assigning an ESCO occupation to each data point, the data was prepared data by removing punctuation, stop words, and other irrelevant words or words that could influence the accuracy of the algorithms (such as the name of companies). Following the pre-processing step was the creation of a TF-IDF (Term Frequency-Inverse document frequency) matrix (Ramos, 2003). 
The resulting matrix had 58 rows (one for each job) and 1531 columns (one for each word/feature considered) was used to test tree machine learning algorithms: (1) Support Vector Machines (SVM) without specific kernel; (2) k nearest neighbors (k-NN) considering a single neighbor as some classes have just two samples, and (3) decision tree with the default behavior of SKLearn toolkit (Pedregosa et al, 2011).

The algorithms performance was measured using the leave one out cross-validation (Berrar, 2019). This is suitable as our dataset is not very large altogether and the imbalance of the data prevented the usage of a k-fold cross validation. Results can be seen in Table 3 with the corresponding confusion matrixes in Figure 4.

Table 3. Accuracies obtained for each machine learning algorithm using leave one out cross-validation.

\begin{tabular}{ll}
\hline Algorithm & Accuracy \\
\hline SVM & 0.810 \\
\hline KNN & $\mathbf{0 . 9 4 8}$ \\
\hline Decision Tree & 0.879 \\
\hline
\end{tabular}

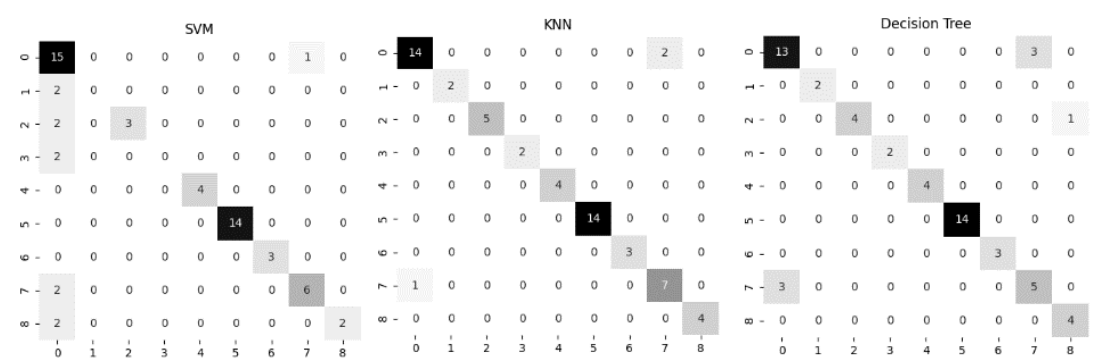

Figure 4. Confusion matrix for each machine learning algorithm. The meaning of each matrix label is in Table 2

\section{CONCLUSION}

This work shows that it is possible to have an accurate overview of the job market dynamics based on the collection of job offerings and by using tools such as the ELK stack to explore and visualize data. This can be done if two issues are considered carefully: (1) have a data model with the features required for a good data description; and (2) when using multiple overlapping sources, as it was our case since a company can advertise the same position in several places simultaneously, an adequate duplicate detection algorithm must be implemented. Here was considered that a similar job proposal in the same month is a duplicate and that after one month is a new job proposal for a similar position. A limitation of this job market overview is that, although this duplicate criterion is adequate for the companies interviewed in previous works (citation omitted due to blind review), we cannot guarantee that all companies have the same behavior and thus the dashboard will present some inaccuracies. However, these inaccuracies are not expected to be meaningful.

The machine learning experiment conducted shows that one can expect to achieve a uniform job description using an ontology such as ESCO. The uniformization of the job description is important for making possible the comparison of the job market dynamics across distinct regions, companies, time periods, etc. Moreover, considering ESCO, this would allow not only look at the job market, the ESCO occupations, but also to the competences more in need by the market, as ESCO relates occupations with qualifications and skills. This implies that with small changes, this platform will be able to show the qualifications and skills more in demand in each region, allowing the adoption of policies to mitigate possible gaps, and thus improving the competitiveness of region. 


\section{ACKNOWLEDGEMENT}

This work was supported by National Funds through the Portuguese Foundation for Science and Technology (FCT_Fundação para a Ciência e a Tecnologia) within IEETA/UA R\&D unit (UIDB/00127/2020) and GOVCOPP (project POCI-01-0145-FEDER-008540), funded by FEDER funds through COMPETE2020 - Programa Operacional Competitividade e Internacionalização (POCI).

"Aveiro STEAM city" project is co-funded by the European Regional Development Fund - ERDF, included in the "Urban Innovative Actions" programme. With a total budget of 6.115.915€ (4.892.732€ ERDF), the project is led by the Municipality of Aveiro in partnership with the University of Aveiro, Altice Labs, Instituto de Telecomunicações, INOVARIA and CEDES.

\section{REFERENCES}

Ahmed Awan, M.N., Khan, S., Latif, K. and Khattak, A.M., 2019. A New Approach to Information Extraction in User-CareerJet (2020). Available at: https://www.careerjet.com/ (Accessed: 30 September 2020)

Centric E-Recruitment Systems. Applied Sciences, 9(14), p.2852.

Berrar, Daniel, 2019, Cross-Validation. In Encyclopedia of Bioinformatics and Computational Biology (Vol. 1, pp. 542-545).

Briscariu, R., 2019. The use of social networks sites in the recruitment process. Management Research and Practice, 11(4), 5-10.

Celik Ertuğrul, D., Karakas, A., Bal, G., Gultunca, C., Elçi, A., Buluz, B., and Alevli, M. 2013. Towards an Information Extraction System Based on Ontology to Match Resumes and Jobs. In undefined (pp. 333-338).

Chen, J., Zhang, C., and Niu, Z. 2018. A Two-Step Resume Information Extraction Algorithm. Mathematical Problems in Engineering, 2018, p.5761287.

Chryssolouris, G., Mavrikios, D., \& Mourtzis, D. 2013. Manufacturing systems: skills \& competencies for the future. Procedia CIRp, 7(2013), 17-24.

ELK (2020) Elastic Stack and Product Documentation. Available at https://www.elastic.co/guide/index.html (Accessed: 30 September 2020)

ESCO (2020) Available at https://ec.europa.eu/esco (Accessed: 30 September 2020)

Freddi, D. 2018, Digitalisation and employment in manufacturing. AI \& SOCIETY, 33(3), 393-403.

Gelpi, A. (2019). Focus on social media as vital element of graduate recruitment campaigns. Recruiting \& Retaining Adult Learners, 21(10), 12-12.

ITJobs (2020). Available at: https://www.itjobs.pt/ (Accessed: 30 September 2020)

Jerman, A., Pejić Bach, M., \& Aleksić, A., 2020. Transformation towards smart factory system: Examining new job profiles and competencies. Systems Research and Behavioral Science, 37(2), 388-402.

Luis Fernández-Sanz, Josefa Gómez-Pérez, and Ana Castillo-Martínez 2017. e-Skills Match: A framework for mapping and integrating the main skills, knowledge and competence standards and models for ICT occupations. Computer Standards \& Interfaces, 51, p.30 - 42.

M. Bajer 2017. Building an IoT Data Hub with Elasticsearch, Logstash and Kibana. In 2017 5th International Conference on Future Internet of Things and Cloud Workshops (FiCloudW) (pp. 63-68).

Pedregosa, F., Varoquaux, G., Gramfort, A., Michel, V., Thirion, B., Grisel, O., ... \& Vanderplas, J. (2011). Scikit-learn: Machine learning in Python. the Journal of machine Learning research, 12, 2825-2830.

Ramos, J., 2003, December. Using tf-idf to determine word relevance in document queries. In Proceedings of the first instructional conference on machine learning (Vol. 242, pp. 133-142).

Szalavetz, A. 2019. Industry 4.0 and capability development in manufacturing subsidiaries. Technological Forecasting and Social Change, 145, 384-395. 